\title{
AGN and starbursts at high redshift: High resolution EVN radio observations of the Hubble Deep Field
}

\author{
M. A. Garrett ${ }^{1}$, T. W. B. Muxlow ${ }^{2}$, S. T. Garrington ${ }^{2}$, W. Alef ${ }^{3}$, A. Alberdi ${ }^{4}$, H. J. van Langevelde ${ }^{1}$, \\ T. Venturi ${ }^{5}$, A. G. Polatidis ${ }^{6}$, K. I. Kellermann ${ }^{7}$, W. A. Baan ${ }^{8}$, A. Kus ${ }^{9}$, \\ P. N. Wilkinson ${ }^{10}$, and A. M. S. Richards ${ }^{2}$
}

1 Joint Institute for VLBI in Europe (JIVE), Postbus 2, 7990 AA, Dwingeloo, The Netherlands

2 MERLIN/VLBI National Facility, Jodrell Bank Observatory, Lower Withington, Macclesfield, Cheshire SK11 9DL, UK

3 Max-Planck-Institut für Radioastronomie Auf dem Hügel 69, 53121 Bonn, Germany

4 Instituto de Astrofísica de Andalucia, CSIC, Apdo. 3004, 18080 Granada, Spain

5 Instituto di Radioastronomia, CNR, Via P. Gobetti, 101, 40129 Bologna, Italy

6 Onsala Space Observatory, 43992 Onsala, Sweden

7 National Radio Astronomy Observatory, 520 Edgemont Road, Charlottesville, VA 22903, USA

8 Netherlands Foundation for Research in Astronomy (ASTRON), Postbus 2, 7990 AA Dwingeloo, The Netherlands

9 Torun Centre for Astronomy, Nicolas Copernicus University ul. Gagarina 11, 87-100 Torun, Poland

10 The University of Manchester, Jodrell Bank Observatory, Lower Withington, Macclesfield, Cheshire SK11 9DL, UK

Received 10 November 2000 / Accepted 14 December 2000

\begin{abstract}
We present deep, wide-field European VLBI Network (EVN) $1.6 \mathrm{GHz}$ observations of the Hubble Deep Field (HDF) region with a resolution of 0.025 arcseconds. Above the $210 \mu \mathrm{Jy} /$ beam $(5 \sigma)$ detection level, the EVN clearly detects two radio sources in a field that encompasses the HDF and part of the Hubble Flanking Fields (HFF). The sources detected are: VLA J123644+621133 (a $z=1.013$, low-luminosity FR-I radio source located within the HDF itself) and VLA J123642+621331 (a dust enshrouded, optically faint, $z=4.424$ starburst system). A third radio source, VLA J123646+621404, is detected at the $4 \sigma$ level. The VLBI detections of all three sources suggest that most of the radio emission of these particular sources (including the dusty starburst) is generated by an embedded AGN.
\end{abstract}

Key words. galaxies: active - galaxies: radio continuum - galaxies: starburst

\section{Introduction}

HST observations of the Hubble Deep Field North (hereafter HDF, Williams et al. 1996) have provided deep, high resolution optical images of the distant universe. These have been vigorously followed up with complementary radio, sub-mm, far infrared, near infrared and X-ray observations (Ferguson et al. 2000 and references therein). The high sensitivity radio observations of Richards et al. 1998 (hereafter R98), Muxlow et al. 1999 (hereafter M99), Richards 2000 (hereafter R00) and Garrett et al. 2000 have thrown new light on the nature of the faint microJy radio source population. These observations, spanning a range of angular resolutions $\left(0.15^{\prime \prime}\right.$ to $\left.15^{\prime \prime}\right)$, suggest that $\sim 60 \%$ of faint sub-mJy and microJy radio sources have radio luminosities, $L_{1.4 \mathrm{GHz}}<10^{23} \mathrm{~W} / \mathrm{Hz}$, steep spectra and angular sizes comparable or smaller than their parent

Send offprint requests to: M. A. Garrett, e-mail: garrett@jive.nl galaxies. In the optical these systems are identified with moderate redshift, star-forming galaxies, often presenting peculiar morphologies and evidence for interactions with other galaxies in the field. The radio emission in these systems arises from a combination of young supernovae, supernova remnants (SNR) and, in particular, relic SNR emission. Approximately $20 \%$ of the faint radio source population are identified with relatively low-luminosity AGN, with the remaining $20 \%$ identified with optically faint sources with no detections down to $I=25.5^{\mathrm{m}}$ in the HFF and $I=28.5^{\mathrm{m}}$ in the HDF (R98, M99, R00). The optically faint systems are thought to be distant galaxies, obscured by dust (Richards et al. 1999). Such systems may only be detectable in the radio and sub-mm where the effects of extinction due to dust are less significant. Since the nature of the optical morphology and optical spectra of these sources is unknown, it is as yet unclear whether individual systems can be identified as pure starburst galaxies, dusty AGN or perhaps some mixture 
of both phenomena. In principle, high resolution VLBI observations can discriminate between these possibilities.

In this paper, we present high resolution, VLBI observations of faint radio sources in the HDF. The aim of these pilot observations, made with the European VLBI Network (EVN), was to probe for the first time, the milliarcsecond (mas) radio structures of the faint microJansky radio source population. Compared with the typical VLBI field of view, the HDF spans a relatively large area of sky and was specifically chosen to be devoid of all strong radio sources $\left(S_{1.4 \mathrm{GHz}}<2 \mathrm{mJy}\right)$. The success of the observations described here relied heavily on the recently enhanced capabilities of the EVN (in particular the introduction of the high sensitivity MkIV data acquisition system), together with the use of the now standard techniques of phase-referencing (e.g. Beasley \& Conway 1995) and wide-field VLBI imaging (Garrett et al. 1999).

\section{EVN observations and data analysis}

Observations of the HDF region were made with the European VLBI Network on 12-14 November 1999 at 1.6 GHz. A total of 32 hours observing time was split into two, 16 hour runs. The EVN included the $100-\mathrm{m}$ Effelsberg (DE), 76-m Lovell (UK), 32-m Medicina (IT), 32-m Noto (IT), 32-m Cambridge (UK), and 25-m Onsala (SE) telescopes; recording data at a total sustained bit rate of $256 \mathrm{Mbits} / \mathrm{s}$ (including both right $(\mathrm{R})$ and left (L) hand circular polarisation). The Deep Space Network (DSN/NASA) 70-m telescope at Robledo (ES) was also used for approximately 11 hours of the total 32 hour run, providing data in one polarisation $(\mathrm{L})$.

The observations were made in phase-reference mode with a typical cycle time of $2.5 \mathrm{~min}$ on the phase-calibrator and $4.5 \mathrm{~min}$ on the HDF. The total on-source integration time on the HDF was $\sim 14$ hours. Two phasecalibrators were used, the primary calibrator J1241+602 (a compact $S_{1.4 \mathrm{GHz}} \sim 455 \mathrm{mJy}$ source lying $2^{\circ}$ from the HDF) and a fainter secondary calibrator J1234+615 $\left(S_{1.4 \mathrm{GHz}} \sim 30\right.$ mJy lying only $23^{\prime}$ from the HDF). This nearby, secondary calibrator was first detected in a targeted MERLIN survey of potential mJy calibrator sources located within $0.5^{\circ}$ of the HDF. Having a nearby calibrator allowed us to reduce possible phase errors associated with ionospheric disturbances (which may have been significant during this period of high solar activity). However, at the time the observations were conducted, the compactness of the secondary calibrator was not known on VLBI scales, it was thus considered prudent to only employ it in every other tape pass, i.e. $\sim 1 / 2$ the total tape passes scheduled.

The data were processed at the NRAO VLBA correlator in Socorro, NM, USA. The phase centre (and telescope pointing position) of the single pass correlation was coincident with a $470 \mu \mathrm{Jy}$ radio source, VLA J123642+621331 (R00), lying just outside the central HDF (in an adjacent HFF). All subsequent data processing was performed with the NRAO AIPS package. The visibility amplitudes were calibrated using the system temperatures and gain information provided by each telescope. The residual delays, fringe rates and antenna gains were determined from $\mathrm{J} 1241+602$, then interpolated and applied to the data. The secondary calibrator was found to have compact structure on EVN angular scales, and thus second order, phase-only corrections were also determined for those tape passes that included the secondary calibrator.

We selected as possible targets, all sources located within $3.5^{\prime}$ of the EVN phase-centre with MERLIN peak flux densities, $S_{1.4 \mathrm{GHz}}$, in excess of $60 \mu \mathrm{Jy}$ (as measured from uniformly weighted maps). Five target sources satisfied these conditions, all of which turned out to be located within $2^{\prime}$ of the phase-centre. A sixth, "blank" field was also chosen, in order to act as a check on the robustness of the imaging process. With the final gain corrections applied, the HDF data were Fourier transformed to the image plane and dirty images were simultaneously generated for the six target fields.

In order to image the entire HDF (an area of $\sim 5 \operatorname{arcmin}^{2}$ ), the HDF data were stored in the form in which the correlator generated them: 512 contiguous $0.125 \mathrm{MHz}$ spectral channels with a visibility averaging time of 1 second. We estimate that for these data, the effects of bandwidth and time smearing are minimal, resulting in no more that a $10 \%$ reduction in source intensity for fields located more than $3^{\prime}$ from the phase centre. A more important limitation on the field-of-view is the fall off of the primary beam response of the larger telescopes in the array (primarily Effelsberg, Lovell and Robledo). The contribution made by these telescopes dominate the naturally weighted images presented here. The most distant target field presented in these observations is located 2 arcmin from the telescope pointing centre. We estimate that our response is down by less than $15 \%$ in fields located 2 arcmin from the phase and telescope pointing centre.

The naturally weighted dirty images are shown in Fig. 1. The images were restored with a Gaussian elliptical beam $\left(26 \times 20\right.$ mas in $\left.\mathrm{PA}=-31^{\circ}\right)$. The $\mathrm{rms}$ noise levels range from $42 \mu \mathrm{Jy} /$ beam (in the field with the strongest detection) to $33 \mu \mathrm{Jy} /$ beam (in a field with no detection). This is higher than the predicted thermal noise $(\sim 23 \mu \mathrm{Jy} /$ beam $)$ which suggests we are currently limited by errors in the calibrator phase solutions. With the maximum side-lobe level at $20 \%$ of the main dirty beam response, CLEANing the 2 fields with clear detections produced no substantial improvement in the image quality.

\section{EVN detections in the central HDF region}

At the $5 \sigma$ detection level, the EVN observations clearly detect VLA J123642+621331 (at the phase centre) and VLA J123644+621133 (located within the HDF itself). Both sources are detected independently in both left and right-hand circular polarisation. The sources are separated by $\sim 2^{\prime}$. There is a $4 \sigma$ detection of J123646+621404, located within 20 mas of the measured MERLIN position. 

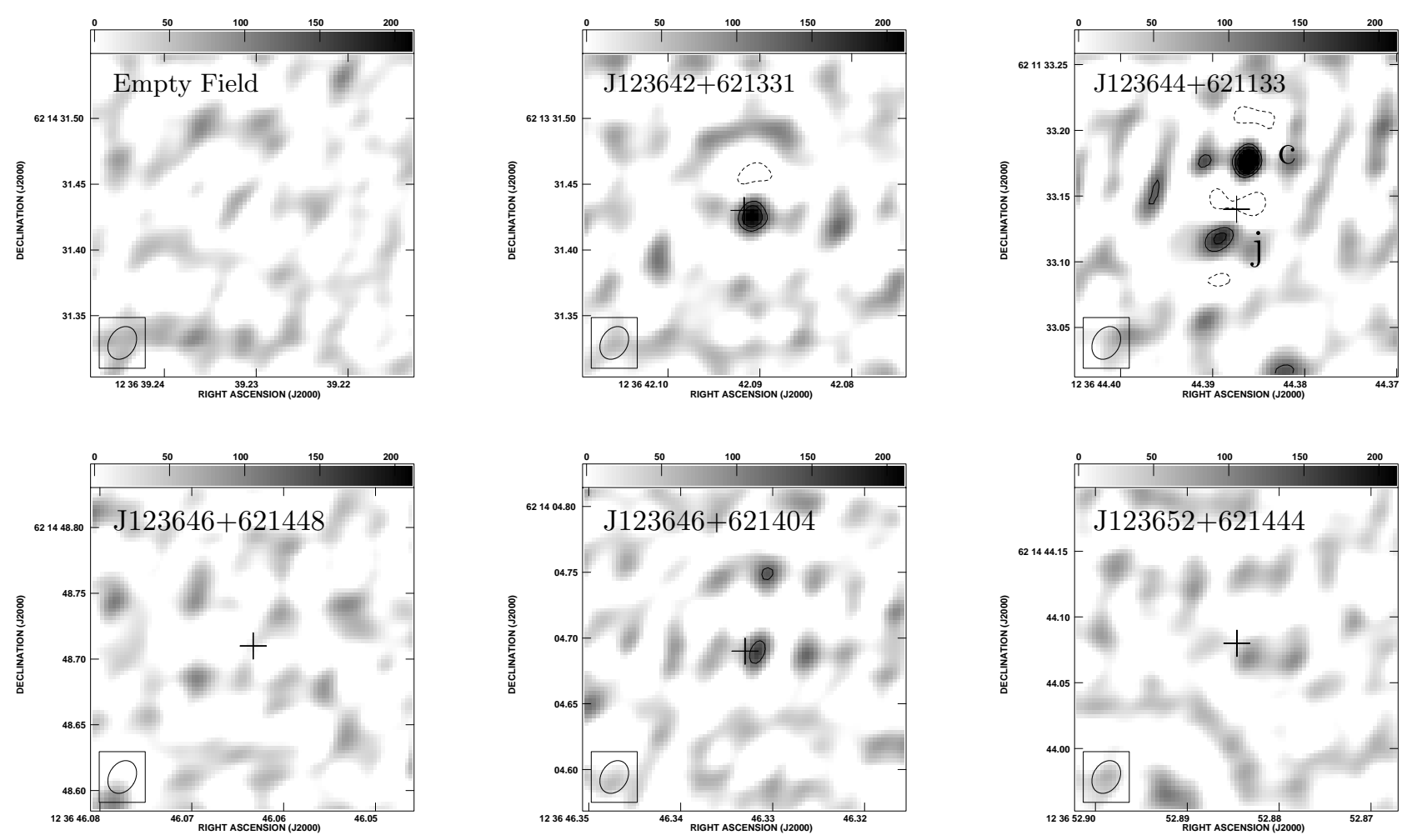

Fig. 1. EVN 1.4 GHz dirty images of the six MERLIN targets. The MERLIN source positions are identified by crosses. Contours are drawn at $-3,3,4,5,6,7,8,9$ times the rms noise level $\sim 40 \mu \mathrm{Jy}$ per beam

Table 1. EVN $1.4 \mathrm{GHz}$ detections in the HDF region

\begin{tabular}{|l|l|l|l|l|l|l|l|}
\hline Source & RA $\left(+12^{\mathrm{h}} 36^{\mathrm{m}}\right)$ & DEC $\left(+62^{\circ}\right)$ & $S_{\text {peak }}$ & $S_{\text {total }}$ & Size & $S_{1.4}$ \\
& $(\mathrm{~s})$ & $\left(^{\prime \prime}\right)$ & $\mu \mathrm{Jy}$ & $\mu \mathrm{Jy}$ & mas & $\mu \mathrm{Jy}$ & $\mu \mathrm{Jy}$ \\
\hline VLA J123644+621133 (c) & $44.38626 \pm 0.0001$ & $1133.1767 \pm 0.001$ & $351 \pm 41$ & $351 \pm 41$ & $\leq 20$ & 1290 & 477 \\
VLA J123644+621133 (j) & $44.38933 \pm 0.001$ & $1133.1162 \pm 0.002$ & $190 \pm 41$ & $214 \pm 77$ & $\leq 30$ & - & - \\
VLA J123642+621331 & $42.09078 \pm 0.0002$ & $1331.4254 \pm 0.001$ & $248 \pm 37$ & $248 \pm 37$ & $\leq 20$ & 467 & 73 \\
VLA J123646+621404 & $46.33177 \pm 0.0002$ & $1404.6893 \pm 0.002$ & $180 \pm 38$ & $180 \pm 38$ & $\leq 20$ & 179 & 168 \\
\hline
\end{tabular}

The EVN source positions, flux densities and sizes of all 3 sources is presented in Table 1 . The 1.4 and $8.4 \mathrm{GHz}$ VLA flux densities (R98, R00) of the sources are also given. Two other target sources, J123646+621448 and J123652+621444, with peak MERLIN flux densities of 60 and $103 \mu \mathrm{Jy}$ were not detected $(\geq 3 \sigma$ level, see Fig. 1). Note that since the primary calibrator $(J 1241+602)$ has an ICRF position, the absolute positions of the sources presented in Table 1 are estimated to be accurate to better than 10 milliarcsecond. These are the most accurate ICRF positions associated with the HDF region.

\subsection{VLA J123644+621133}

VLA J123644+621133 is identified with a bright $(I \sim$ $\left.21.05^{\mathrm{m}}\right) z=1.013$, red elliptical galaxy (R98). The radio source is clearly identified by its large scale radio structure and luminosity as an FR-I (R98, M99). The EVN (see Fig. 1) detects a compact core component (c) with an estimated size $<20$ mas, implying a brightness temperature, $T_{\mathrm{b}}>410^{5} \mathrm{~K}$. We also detect an additional $5 \sigma$ component $(\mathrm{j})$ located $\sim 60$ mas south of the core component. Presumably this source $(\mathrm{j})$ is a knot in the extended VLA/MERLIN jet that also trail southwards of the core in a similar position angle. Note that the MERLIN position for this source (see Fig. 1) is strongly affected by the extended jet structure, lying mid-way between the compact core (c) and the knot in the jet (j). The VLBI structure observed in this source, supports the view (M99) that the rare "classical" radio sources detected in the HDF and $\mathrm{HFF}$, are the mJy tail of the AGN radio source population. It is worth noting that VLA J123644+621133 is probably an order of magnitude more distant than any other classical FR-I radio source so far studied with VLBI. 


\subsection{VLA J123642+621331}

VLA J123642+621331 lies just outside the HDF, in an adjacent HFF. At radio wavelengths it is a relatively bright, steep spectrum radio source $\left(S_{1.4 \mathrm{GHz}} \sim 470 \mu \mathrm{Jy}\right)$ with no optical counterpart to $I \leq 25^{\mathrm{m}}$ (R98). Deep HST NICMOS $1.6 \mu \mathrm{m}$ imaging clearly detects an extremely red $23.9^{\mathrm{m}}$ counterpart to the radio source with a disk profile, and spectra obtained by the Keck II telescope show a single strong emission line, which is identified with Ly $\alpha$ at $z=4.424$ (Waddington et al. 1999, hereafter W99). VLA J123642+621331 is also identified in the ISO supplementary list of sources $\left(S_{15 \mu} \sim 23_{-12}^{+10} \mu \mathrm{Jy}\right.$, Aussel et al. 1999) but there is no $850 \mu \mathrm{m}$ sub-mm SCUBA detection to a limit of 2 mJy (Hughes et al. 1998). W99 interpret the source as a dust obscured, nuclear starburst with an embedded AGN.

The high resolution $0.15^{\prime \prime} 1.4 \mathrm{GHz}$ VLA-MERLIN observations (M99) resolve VLA J123642+621331, showing that $10 \%$ of the flux density resides in an extended component lying to the east of an unresolved core. It is the core which is detected by the EVN at $1.6 \mathrm{GHz}$. No other sources of radio emission $(>3 \sigma)$ are detected in a $0.7^{\prime \prime} \times 0.7^{\prime \prime}$ field centred on the core. The main question is whether the radio emission arises due to star-formation processes or AGN activity. The EVN limit on the measured core size $(<20$ mas) implies a brightness temperature, $T_{\mathrm{b}}>210^{5} \mathrm{~K}$. While this in itself does not rule out a starburst interpretation for the radio emission, the high luminosity $\left(\sim 10^{25} \mathrm{~W} / \mathrm{Hz}\right.$ i.e. $\sim 100$ times more luminous than the ultra-luminous starburst Arp 220) argues strongly for an AGN interpretation for most of the radio emission. Further, high resolution, global VLBI observations are required to confirm an AGN interpretation for this radio source.

\subsection{VLA J123646+621404}

VLA J123646+621404 is a flat spectrum radio source located within the HDF itself and is associated with a very red, face on, $z=0.960$ spiral galaxy (R98). Rowan-Robinson et al. (1997) interpret the ISO $7 \mu \mathrm{m}$ emission in terms of a massive starburst but the presence of broad MgII emission (Phillips et al. 1997), radio variability (R98) and X-ray emission (Hornschemeier et al. 2000) suggest the spiral is host to an AGN. We see a $4 \sigma$ radio source in this field that is coincident with the MERLIN position (see Fig. 1). We suggest this is compact radio emission from the AGN.

\section{Conclusions}

The results presented here clearly demonstrate the potential for VLBI imaging of the micro-Jansky source population. We have reliably detected two radio sources within the HDF region. One of these VLA J123644+621133 shows all the typical properties of a low luminosity, classical FR-I radio source. The second, perhaps more interesting source is VLA J123642+621331. Our detection of a relatively compact but high luminosity radio core, further supports the suggestion by W99 that this is a dusty, starforming galaxy with an embedded AGN. We also appear to have detected radio emission from the AGN associated with VLA J123646+621404.

Global VLBI observations with 3 times the resolution and 4 times the sensitivity of the pilot observations presented here, are now achievable. With a linear resolution of $\sim 30 \mathrm{pc}$ at cosmological distances, only Global VLBI can resolve nuclear star-forming regions like those observed in Arp 220 (Smith et al. 1998). Targeted observations of the optically faint radio source population (of which VLA J123642+621331 is just one example) may throw new light on the nature of the associated population of dusty sub-mm sources, recently discovered by SCUBA (Hughes et al. 1998). In the absence of near-infrared, optical, ultraviolet and even X-ray emission (due to dust obscuration), future VLBI observations will be a crucial diagnostic in distinguishing between AGN and starburst activity in these systems.

Acknowledgements. We would like to thank the staff of the EVN observatories who made these observations possible. We also thank Lorant Sjouwerman (JIVE) and the staff of NRAO for supporting the correlation of these observations. We thank M. Eubanks (USNO) for obtaining an ICRF position for J1241+602. The National Radio Astronomy Observatory is a facility of National Science Foundation operated under cooperative agreement by Associated Universities, Inc.

\section{References}

Aussel, H., Cesarsky, C. J., Elbaz, D., \& Starck, J. L. 1999, A\&A, 342, 313

Beasley, A. J., \& Conway, J. E. 1995, in PASPC 82, ed. J. A. Zensus, P. J. Diamond, \& P. J. Napier, 328

Ferguson, H. C., Dickinson, M., \& Williams, R. 2000, to appear in ARA\&A, 38 [astro-ph/0004319]

Garrett, M. A., Porcas, R. W., Pedlar, et al. 1999, NewAR, 43, 521 [astro-ph/9906108]

Garrett, M. A., de Bruyn, A. G., Giroletti, M., et al. 2000, A\&A, 361, L41 [astro-ph/0008509]

Hornschemeier, A. E., Brandt, W. N., Garmire, G. P., et al. 2000, ApJ, 541, 49

Hughes, D. H., Serjeant, S., Dunlop, J., Rowan-Robinson, M., et al. 1998, Nature, 394, 241

Muxlow, T. W. B. Wilkinson, P. N., Richards, A. M. S., et al. 1999, New Astron. Rev., 43, 623

Phillips, A. C., Guzman, R., Gallego, J., et al. 1997, ApJ, 489, 543

Richards, E. A. 2000, ApJ, 533, 611

Richards, E. A., Kellermann, K. I., Fomalont, E. B., et al. 1998, AJ, 116, 1039

Richards, E. A., Fomalont, E. B., Kellermann, K. I., et al. 1999, ApJ, 526, L73

Rowan-Robinson, M., Mann, R. G., Oliver, S. J., Efstathiou, A., et al. 1997, MNRAS, 289, 490

Smith, H. E., Lonsdale, C. J., Lonsdale, C. J., \& Diamond, P. J. 1998, ApJ, 493, L17

Waddington, I., Windhorst, R. A., Cohen, S. H., Partridge, R. B., et al. 1999, ApJ, 526, L77

Williams, R. E., Blacker, B, Dickinson, M., et al. 1996, AJ, 112, 1335 